emphasizes the particulate nature of music and language - both assembled from discrete elements - and suggests that these two domains may share a set of brain structures. By contrast, studies of brain lesion data (from patients with deficits that follow specific brain damage) and brain imaging results are also consistent with a view that music and language processing are, at least in part, segregated.

Patel thinks that there are more general, perhaps computational, links between the two. For example, when discussing rhythm, he proposes that the processing of nonperiodic signals is similar in both music and speech. In the section on syntax, he argues that the brain uses similar neural resources to integrate the hierarchical organization of music and language. When discussing evolutionary and developmental similarities, the notion of 'beat-based rhythm processing' emerges as a crucial feature that may underlie music and speech.

Patel's perspective is laudably cross-linguistic and multicultural, citing extensive work from non-Indo-European languages and non-Western-based musical systems. On the website accompanying the book (http://tinyurl. $\mathrm{com} / 2 \mathrm{z} 2 \mathrm{cve}$ ), Patel provides stimulating sound and video examples that clarify the phenomena described in each chapter.

Music, Language, and the Brain is much more than a textbook by one of the field's most influential practitioners. Each chapter can serve as a stand-alone monograph, and can be read at many levels. There is enough clarity for the general reader to follow the lines of argument, while the specialized reader will discover Patel's sophisticated and well-researched positions. Ideal for students of music cognition and language, the book outlines numerous experiments and hypotheses - many unusual - that draw together psychology and neurobiology.

If one can criticize anything, it is that Patel's discussion of the neurobiological foundations of auditory cognition is less nuanced and inspirational than his treatment of behavioural research. That said, we know little about the neuronal bases of complex psychological phenomena. Our understanding of auditory cognition is still mostly informed by behaviourally based psychological research, and in that domain, Patel's discussion is second to none.

In this definitive analysis of music cognition and its relationship to language, Patel gives us a work of exceptional scholarship and clarity. Much needed, it elevates the discussion to a level that these exciting areas merit.

David Poeppel is a professor of linguistics and biology and Elika Bergelson is a Baggett research fellow at the Linguistics Department, University of Maryland, College Park, Maryland 207427505, USA.

\title{
Catching a ride on sunshine
}

\section{Solar Sails: A Novel Approach to \\ Interplanetary Travel \\ by Giovanni Vulpetti, Les Johnson and \\ Gregory L. Matloff \\ Springer: 2008.250 pp. $€ 16.50$}

\section{Stuart Clark}

Conceptually simple and romantic, solar sailing is an enchanting technological solution for space exploration. When a large reflective sail is unfurled in space, photons of sunlight collide with the sail fabric, imparting pressure and causing the sail to move. Such photons are not the electrically charged particles that constantly flow from the Sun to create the solar winds, they are the actual sunlight itself. The angle of the sail to the Sun and its direction of travel determine whether a propelled craft speeds up or slows down, just as a yacht changes course on the sea.

Solar Sails: A Novel Approach to Interplanetary Travel is the latest book to explore this topic, one that has been tackled only a handful of times in the past 20 years. Aimed at undergraduates, the book convincingly captures the history of ideas about solar sails, their current state of play and their future promise.

Moving according to the constant interplay of gravity and the pressure of sunlight, spacecraft pushed by solar sails are highly manoeuvrable. They can skate along unusual interplanetary trajectories that traditional point-and-shoot rocket-propelled craft would find difficult, if not impossible, to navigate. In the flexibility stakes, the only current competition is from the newly tested but expensive ion-drive engine that powers the SMART-1 Moon mapper built by the European Space Agency (ESA) and NASA's Deep Space One asteroid probe. These propulsion modules run by expelling charged particles, or ions, and can operate using less fuel than standard chemical engines; however, they are technologically trickier and thus expensive to build.

The idea that sunlight exerts pressure has been around for more than a century, since physicist James Clerk Maxwell proposed it in the 1860s. In the 1970s, metre-long solar sail fins - rather like the fins on a 1950s American car - were attached to the Mariner 10 Mercury space probe to adjust its alignment. Today, some satellites are steered with small sail vanes, a technology patented by the aerospace company EADS Astrium. The extra force of sunlight is a hindrance when fine control of movement is required, as with the next generation of formation-flying spacecraft in ESA's proposed Darwin interferometry mission to search for life on extrasolar planets. Such vessels must instead be designed to minimize displacements or, at least, to all suffer the forces equally.

Despite the opportunities, solar sails have yet to be used for propulsion in space. The pressure of sunlight is so slight that a vast sail area would be needed to carry a worthwhile payload of instruments through space. Deploying such a sheet presents an equally vast challenge, and has remained the solar sailor's Achilles' heel.

With useful sails being many tens to hundreds of metres long, these mighty structures must be packed into the equivalent of a suitcase for launching and then faultlessly unfurled once in space. If the sail snags, tears or fails to deploy, the mission is over. This risk deters many potential users; according to one project scientist at the ESA: "Why jeopardize your science by relying on an untested technology?"

Scientists and space agencies have, until recently, been resistant to solar sailing. This negative attitude was reinforced by the failure of the Planetary Society's Cosmos-1 sail, launched atop a converted Russian intercontinental ballistic missile on 21 June 2005 from a submarine in the Barents Sea north of Russia. The upper-stage rocket motor failed,
The Infinite Cosmos: Questions from the Frontiers of Cosmology

by Joseph Silk (Oxford Univ. Press, E9.99)

Summarizing the latest thinking on the Universe and its fate, Joseph Silk muses on the scientific discovery process and the history of ideas about the cosmos. "Black holes, galaxy formation, dark matter, time travel, string theory and the cosmic microwave background all get a mention" (Peter Coles, Nature 441, 285; 2006).

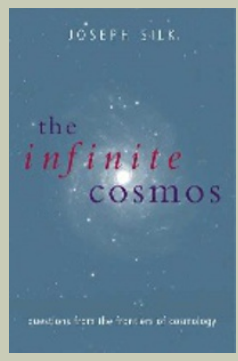

King of Infinite Space: Donald Coxeter, the Man Who Saved Geometry by Siobhan Roberts (Profile Books, $£ 14.99$ ) Donald Coxeter helped to bring geometry back into the mainstream at a time when it was unpopular even with mathematicians. Siobhan Roberts' biography describes his personal and professional life and shows how his impact can be felt in architecture, cosmology, crystallography, immunology and more.

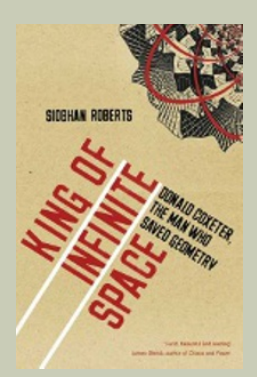




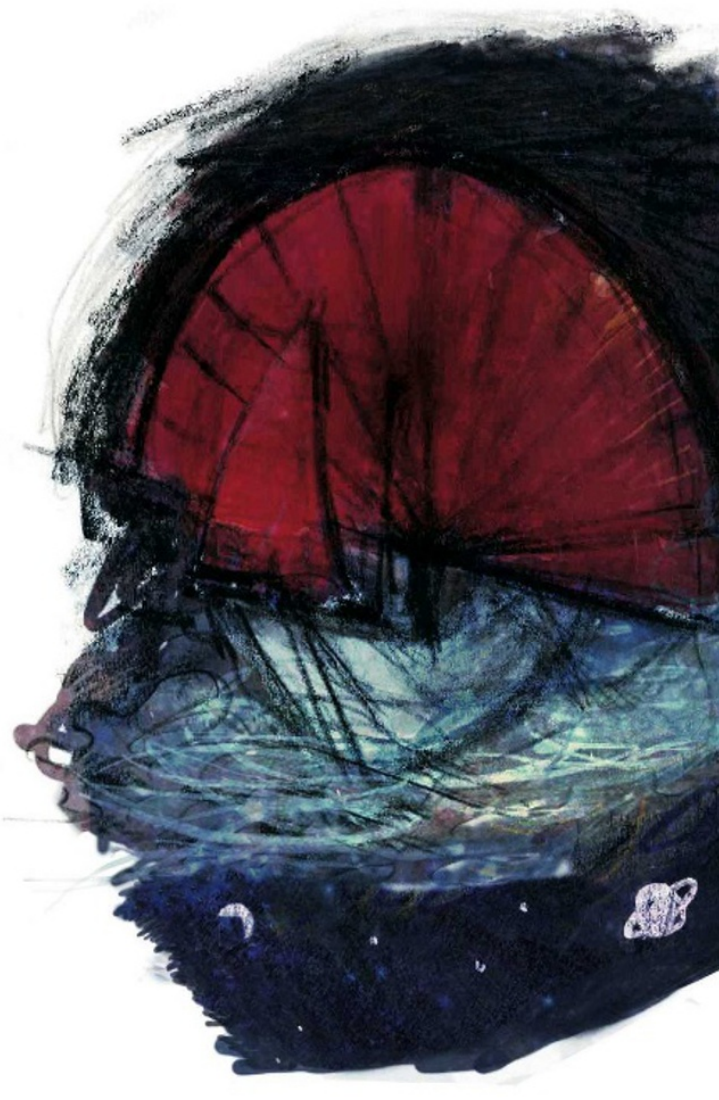

dooming the mission to failure before the sail mechanism could even be tested. Although the test was inconclusive, the perceived lack of success reflected badly on the solar-sail initiative itself.

Now the tide is beginning to turn. Groundbased tests in Europe and the United States have successfully deployed sails of about 20 square metres thanks to improvements in sail-opening mechanisms. The German Aerospace Centre has used plastic booms reinforced with carbon fibre, and NASA has used inflatable booms that harden when exposed to the coldness of space. Even more impressively, the Japanese space agency JAXA has carried out two successful sub-orbital deployment tests. Made of reflective films 7.5 micrometres thick and some 10 metres in diameter, the sails were flown to an altitude of 122 kilometres, where one opened up like a clover-leaf, the other like a fan. JAXA followed this up two years later in 2006, with a successful 20-metre-wide sail deployment from a balloon at an altitude of 35 kilometres.

Some space missions can be performed
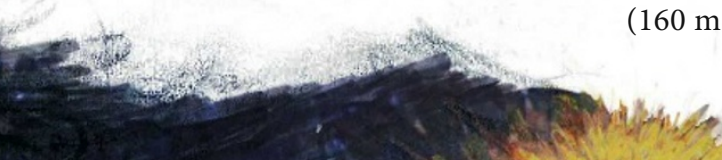

A
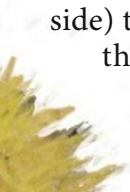

holds the sail in space, it can be angled so it hovers like a kite over the poles of a planet, making solar-sail craft ideal anchors for communications and remote-sensing satellites.

Of course, there are limitations. Solar sails lose their power and manoeuvrability when they are far from the Sun, out beyond Jupiter. They are also unable to assume low orbits around planets with atmospheres because the sails are susceptible to drag.

Suitable for aerospace students and keen enthusiasts alike, this book may one day inspire some of them to build a solar-sailpowered vessel. Although there is still a long way to go, this useful volume will help speed up that day. with solar sails Placing a satellite in a polar orbit around the Sun using a rocket requires a large expenditure of energy, and hence fuel. A craft propelled by a solar sail would take only five years to fly there from Earth but would require a huge sail area of 25,600 square metres

Stuart Clark is a visiting fellow at the Centre for Astrophysics Research, University of Hertfordshire, Hatfield, AL10 9AB, UK. He is author of The Sun Kings.

\section{Imaging the unseen}

\section{Six Stories from the End of \\ Representation: Images in Painting, \\ Photography, Astronomy, Microscopy, \\ Particle Physics, and Quantum \\ Mechanics, 1980-2000 \\ by James Elkins \\ Stanford University Press: 2008. \\ 320 pp. $\$ 65$}

\section{Felice Frankel}

In Six Stories from the End of Representation, James Elkins makes a brave and laudable attempt to address in parallel the communication of ideas in both the sciences and the humanities.

"I believe that the clearest, most fruitful response to the abyss between the humanities and the sciences is to set out the disciplines, in detail, side by side, and let them tell their stories in their own languages. As far as I can see, that is the only way to produce a book that can be read by scientists and humanists without the creeping feeling that their disciplines are better explained - or explained away - by someone who does not really understand them," he writes.

Elkins' approach struck me as exciting. A respected and prolific scholar, he promised a view of scientific images distinct from that taken by other art historians. I was eager to see the images to which he was going to introduce us, and how they were to "tell their own stories". After all, Elkins declared that "the images came first" in preparing the book, and that the images "are among the best that have been made in the last several decades".

\section{Science of Describing: Natural History in Renaissance Europe}

by Brian W. Ogilvie (Univ. Chicago Press, $\$ 27, £ 14$ ) In the mid-sixteenth century, naturalists developed tools for observing and describing nature, enabling them to assess and share their findings with others. Interpreting this change over four generations, Brian Ogilvie "has written the story of how science constantly reinvents itself, seen through the lens of the pre-linnaeans", wrote Sandra Knapp (Nature 442, 871; 2006).

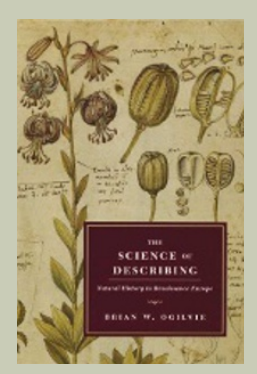

\section{Digital Art (Revised Edition)}

by Christiane Paul (Thames \& Hudson, E8.95)

The digital format offers new possibilities for artistic expression. In an updated volume, Christiane Paul addresses how viewers interact with such works, and explores links with artificial life and intelligence, activism and networks, as well as the collection, presentation and preservation of digital art.

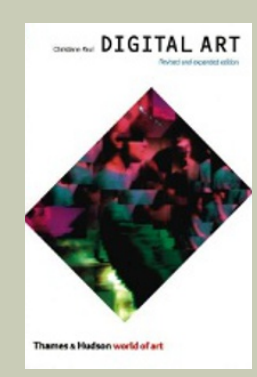

